\title{
A New Study in Support-Driven Middleware for VANET
}

\author{
Phool Singh Gour \\ Department of Computer Science Engineering \\ Oriental Institute of Science and Technology \\ Bhopal India
}

\author{
Amit Shrivastava \\ Department of Computer Science Engineering \\ Oriental Institute of Science and Technology \\ Bhopal India
}

\begin{abstract}
Vehicular Adhoc Network (VANET) can be used as quite a few apps for instance environmentally friendly keeping track of, commercial infrastructure safety, health care apps, along with targeted visitors handle. The look and continuing development of this kind of purposes should address quite a few problems set through VANET features on just one give as well as the specific programs on the other half. Certainly one of the emerging methods for soothing most of these challenges will be exploitation overhaul-focused middleware (SOM). Service-oriented computing, generally, is designed to create services usable and readily available by means of standardized types along with practices without having to worry regarding the underlying infrastructures, growth models, as well as enactment details. A little could perform an important role within aiding the planning, progress, along with implementation and services information-orientated techniques. This may help reach interoperability, reduce pairing, in addition to heterogeneity support. What is more, SOM solutions will certainly supplying no-practical needs such as scalability, reliability, flexibility, and Quality of Services (QoS) assurance. That report surveys the current work in SOM and also the developments and also difficulties to become addressed when coming up with and establishing these kinds of solutions for VANET.
\end{abstract}

\section{Keywords}

VANET , middleware , QoS , SstreaMWare

\section{INTRODUCTION}

VANET purposes have got received a substantial energy by way of your innovations associated with technological knowhow inside dispersed processing, marketing, Wi-Fi communication theory, detector technology, in addition to inlayed devices. These kind of developments provided a wonderful chance for possessing a variety associated with VANET purposes similar to product traces settings, transportation, polluting of the environment supervising, devastation deterrence, commercial infrastructure security, home ground checking, rural health and fitness overseeing, along with targeted visitors command, which can be important for the actual improvement of different job areas these equally remedies, natural environment security, creation, executive, and also organization. Generally, these kinds of apps are created employing unlike heterogeneous products like large-finish hosts, RFID elements, cellular phones, safe-keeping products, sensors, and spiders. The product communicate via many different Wi-Fi/pumpedup(a) networking engineering. Inside improvement, the units are generally manipulated by a few application elements produced by unique vendors and executed employing various development different languages in addition to products. As a result of high distribution, powerful qualities, along with heterogeneousness in the gadgets, communication technologies and also application parts applied; employing these kinds of purposes can be low-unimportant. You'll find typically integration, scalability, stability, security, serviceability, QoS, and functional troubles to get deemed. Including a middleware covering is really a book way of seek to meet the design and also execution problems associated with VANET programs. You can find unique middleware approaches intended for VANET [27]. This kind of methods offer critical operates many different applications including effective application installing along with facts gathering or amassing. Nonetheless, tough one characteristic are expected within the middleware to lessen the complexness in the design and style and also enactment with the needed application for several VANET applications.

The most current solutions that offer in high spirits flexibleness with regard to adding fresh along with state-ofthe-art functions to help VANET middleware could be the program-focused approaching. Program-oriented middleware (SOM) realistically vistas VANETs as being a service agency regarding end user applications. SOM delivers abstractions for your sophisticated underlining VANET via a set of solutions required by VANET purposes. This type of services is usually information accumulation, adaption, security, personal-corporation, reliability, and management providers. All these and also other advanced services is usually designed, carried out, and also integrated in the SOM fabric use a flexible and atmosphere to develop good VANET purposes. Some research work is actually accomplished about surveying these different facets associated with middleware along with development paradigms with regard to VANET. As an example, [26] reviewed several middleware initiatives for VANET; diverse middleware issues as well as approaches intended for VANET were being interviewed throughout [27]; as well as [18] shown and also classified digital machines for VANET. Moreover, [46] surveyed the actual programming designs for sensing element communities, while the state of the art with encoding techniques pertaining to VANET had been surveyed throughout [38]. This kind of analysis attempts are for general encoding in addition to middleware pertaining to sensing unit networks. On the other hand, none of the present work looked into the actual current state regarding inquiry within the style as well as growth and development of SOM pertaining to VANET. In this particular papers, all of us assessment in addition to go over unlike methods involving SOM pertaining to VANET, in addition to existing some of the challenges that they encountered. Therefore, within Section 2, this site offers some background information along with associated aspects. Portion 3 looks at the overall middleware issues regarding VANET. The actual SOM specifications regarding VANET are outlined in Area 4 and also the actual SOM methods intended for VANET are generally reviewed in Segment 5. In section 6 provides a treatment from the categories of diligence according to his or her various wants for middleware services. A part 7 supply a talk with the SOM challenges regarding VANET, as well as Portion 8 concludes this cardstock. 


\section{BACKGROUND AND CONCEPTS}

Not long ago, overhaul-driven computing (SOC) $[9,25]$ has become a preferred design on the more common monolithic along with node/machine advancement products. The idea triumphs over several issues as well as troubles the two of these kinds rich person faced. SOC offers a encouraging method for enabling computer software suppliers to provide the software applications since products and services. An excellent some sort of fabric to be able to signify business operations seeing that unbiased adventures (providers) having distinct and also available interfaces. The communications amongst solutions are executed by using a stock description words like XML, that makes it easy to desegregate various services to construct a profitable business application along with address problems related the integrating connected with heterogeneous programs inside a spread natural environment. Companies can certainly follow through 1 enterprise physical process or possibly a pair of various processes which have been presented for plug-in to heterogeneous products and services. Providers might be highly-developed having a massive amount technology, such as SOAP, Relaxation, RPC, DCOM, CORBA, in addition to Net Products and services. The general style - supportorientated structures (SOA) - generally entails iii briny players: your program service, your program dealer, plus the services requester. The supplier types along with advances an email finder service. This overhaul specialist creates this service available to the rest of earth through general public registries such as General Description Discovery and Is intergrated (UDDI) for world wide web companies. The actual program suppliant locates this synonyms inside the public pc registry along with adheres while using service agency to be able to invoke the web providers essential. There are several people (analysts in addition to providers) using a number of levels of understandings as well as uses involving service oriented computing. As outlined by [40], the particular SOA tactic helps make the following issues achievable:

- Several apps making within various platforms can certainly effectively talk to 1 another

- Software program services are offered to absolve-consumer apps through publicized/ascertainable connects

- Solutions are loosely packaged as a result cutting down dependencies as well as assisting reuse

- Overhaul callers usually are in addition to solutions needed for your continuance in the usage alone (p)

- A new partnership is created among the players: Overhaul Company, discovery firm, along with assistance suppliant

- Connections relating to the contributors include this publish, come across along with emergency procedures

- Overhaul shoppers targeted-certain services via distinct connects that are uncovered from the services computer registry and development elements Middleware, on the whole, provides capabilities in order to desegregate and also recycling software package components on demand and helps abstractionist this dispersion and heterogeneity from the fundamental processing natural environment and also providers readily available. In addition, it supports the summation regarding no-practical prices for instance interoperability, load balancing, scalability, reliability, availability, usability, extensibility, manageability, reusability, services discovery, Service quality (QoS) stability, efficiency, autonomocity, and security. Several middleware platforms were developed to provide brand new ideals for several methods like venture programs [11], clustering processing [2], cellular random sites [10,28], as well as robotics[37]. The particular elements and also solutions are usually depending on the reuse regarding present procedures, practices, software program, in addition to devices to add your needful prices. You will find various middleware strategies intended for VANET. Most of these methods will be the virtual device, database, software pushed, and also message-focused middleware [35]. Your virtual device strategy consists of interpreters and also cellular requirements printed in quests that will stand for this application and are also viewed from the exclusive equipment. The particular organization inserts in addition to directs the quests with the net utilizing tailored formulas for instance one particular pertaining to and minimize boiler suit power using up. Inside databases approaching, the actual full network can be regarded as some sort of electronic database program. This approach provides a light-to help-utilize screen which allows anyone issue inquiries on the warning community to excerpt this believed information of interest. The application form influenced method delivers a great architectural mastery of which extends to the actual system project collection. This specific allows coders for you to okay-song the actual network around the base of request requirements for example $\mathrm{QoS}$, configuration, and management. Although, this process offers outstanding performance strengths, it may give you a specific middleware that may be snugly in addition to the application rather than a normal determination middleware. Finally, what it's all about-orientated middleware technique offers publishsupport components for you to aid information trade between the sensors as well as the drain nodes. The effectiveness of this specific image lies in its help for asynchronous conversation that can help in preserving electricity inside the particular VANET. This method can be quite suited to VANET exactly where nearly all applications are based on events. The actual middleware methods for VANET offer some `significant capabilities many different purposes like useful application installation in addition to efficient information gathering or amassing. On the other hand, more complex features are required within the middleware to relieve the complexity involving designing as well as employing application a variety of VANET software. Examples of these types of superior capabilities are usually edition, safety measures, ego-group, management, QoS, reliability, context-awareness, and management. There are a few prosperous attempts to develop middleware programs for VANET to offer a single or maybe few of these advanced functions. However, many of these are considered specialized middleware websites that is finest used for some kinds of purposes as well as. This is because they will supply limited operates, although most VANET applications normally will need many as well as almost all of the probable superior characteristics. Applying SOM to support solutions exploitation and also utilization have been strongly suggested by means of several experts which necessitate applying overhaul-oriented middleware (SOM) all over traditional middleware of which links divers parts along with methods and offers multiple-sales channel entry to services, by way of fluid in addition to mobile-products along with sensor units on the assortment connected with networks including the Cyberspace, UMTS, XDSL, and wireless [41]. In a different facet, the particular creators of these studies [36] place certain requirements in addition to difficulties associated with SOM intended for pervasive surroundings. One of several requirements, you will find principal prerequisites 
for instance content exchange, ability to move help, stability along with overhaul verbal description, breakthrough discovery, as well as control. There's also cross-cutting needs including QoS support, liberty and also circumstance awareness. Hence, latest work rich person precise the usage of SOM with regard to VANET to produce high freedom regarding putting brand new characteristics to help VANET middleware. Contrary to other methods, unexampled operates might be extra for the middleware simply by introducing unexampled products and services. SOM of course views VANET to be a service agency with regard to end user purposes. SOM provides abstractions for your complex underlying VANET by using a number of advanced services required by VANET apps. These types of services is usually made, applied, along with very easily built-in within the SOM to provide a accommodating and easy environment in order to develop VANET software.

\section{MIDDLEWARE CHALLENGES FOR VANET}

VANET middleware is usually a software system infrastructure which glues with each other the sensor multilevel electronics, operating systems, system piles, along with programs. A whole middleware result really should include a runtime environs which sustains and also harmonizes numerous apps and also standardized system services for example files collection, handle, and also supervision procedures which could adjust to butt software. With add-on, it must consist of mechanisms to realize adaptive, risk-free, and also successful system resources utilization to extend your sensing unit community lifestyle. The actual VANET middleware bed covering the complexness in the community surroundings aside separating the app from the conversation along with ironware particulars.

The structure and also growth of an effective middleware level for VANET isn't unimportant. It has to take care of a lot of troubles influenced from the VANET traits on one hand in addition to the actual applications require on the other guitar. Next is often a talk of those troubles:

\subsection{Ironware Methods}

The arrival within microelectronics technological know-how made it achievable to design miniaturized equipment inside order associated with cubic $\mathrm{cm}$ conclusion also littler. Constrained with vitality as well as individual methods, these kind of small units may very well be started inwards lots or perhaps hundreds in unpleasant and also unpredictable environments, exactly where in some cases not any physical contact could possibly be feasible for alternative or even almost any care opportunity; consequently, Wi-Fi interaction could be the only way intended for rural approachability. Some sort of middleware must provide elements for an efficient standby time with the processor chip and memory even though enabling decrease power interaction. A warning client really should achieve it is several standard surgical procedures (realizing, facts running, and also transmission) while and minimize assets usage avoiding rapid means weariness. To illustrate muscularity informed middleware, it ought to be able to force most of the products elements like the radio stations are likely to be turned off most associated with the time in support of initialize them with respect to the application requires.

\subsection{Alterations in Topology and Dimension}

The actual topographic anatomy with the community is definitely be subject to frequent modifications because of different factors including product disappointment, product ability to move, relocating obstacles, along with interferences. If the request will get larger, this multilevel should be whippy sufficient to help encourage the inclusion connected with additional nodes anywhere at any time without having poignant net execution. Furthermore, in the event several nodes or hyperlinks go bad, your middleware can statement the particular failing in addition to take automated steps with the idea to right the issue or even carry option actions to keep up multilevel functions till your inability is preset. Likewise, middleware really should help systems for personal-configuration as well as self-repair of libraries regarding detector nodes. On top of that, your middleware should be able to alter circle gadgets qualities like granny varies as well as snooze durations to sit in just about any modifications in this topology.

\subsection{Heterogeneousness}

Mentioned previously before, inside a VANET, equipment, hyperlinks, software factors might be heterogeneous as well as involve particular encoding types in addition to resources make use of and incorporate these people inside of a VANET request. Thus, the middleware must provide a number of things to meet the major concern involving bridging this break between hardware technologies fresh probable as well as the wide essential pursuits. It should create technique elements interfacing using the different kinds involving equipment in addition to networks, solely held by basic distributed simple operating-system abstractions. Consequently, this specific should make that easier to produce the software when using the circle without being concerned regarding the minimal-stage facts.

\subsection{Net Corporation}

Contrary to standard networks, indicator networks have to handle resources - strength, bandwidth, and also producing power that are dynamically modifying. Another essential difficulty inwards sensing unit systems is usually to documentation very long-working applications, in which efficient pattern regarding course-plotting standards are essential to permit your community may manage provided that doable. Advertisement hoc net resources development process should be supplied, given that know-how on the system is critical for any multilevel to function effectively.

Some sort of detector node must understand its very own localization inside community besides the solid topology. Several significant difficulties in method parameters such as community size of it and density for each sq mile influence your tradeoffs in between response time, trustworthiness, and ingestion. A middleware must offer the sturdy operation regarding warning networks regardless of these types of character through changing to this adjusting network natural environment.

\subsection{App Understanding}

Another critical in addition to one of a kind property or home involving middleware for VANET is actually dictated through the pattern guidelines associated with application information. Nevertheless, middleware has to include systems regarding inserting program information inside structure of the VANET. It will allow mathematical function app transmission requirements in order to net guidelines that permit very good-tuning in the circle overseeing procedure. 
E.g., different software possesses unlike stability specifications and also a fixed is through not suitable for many programs. Hence, the actual middleware should be able to choose the know-how about your tolerable stability ranges in the app along with chart this needed features to help the actual commercial infrastructure to maintain it. One more illustration is in the array of protection exactly where a number of applications deal with very large geographic parts along with need wide-selection conversation; while others are utilize manner heavy systems thence call for quickerassortment connection. The middleware services should be able to adjust these kinds of wants and modify sign stages depending on each and every program specifications.

\subsection{Security Measures}

VANET are commonly implemented in internet domain names which entail delicate facts and also essential services such as army, professional medical, and also recovery services. Such areas, it will be important in order to safe transmission, overhaul delivery, along with overhaul access. Moreover, the large deployment regarding VANET with abrasive circumstances increases their own contact with harmful assaults like self-abnegation and services information along with dodgy nodes. Moreover, the particular Wi-Fi average encourages eavesdropping as well as adversarial bundle shot for you to skimp the particular network operate. All these factors make the idea essential to aid stability services regarding SOM with regard to VANET. In such cases encrypted shield services are needed to obscure critical information, VANET monitoring companies are essential to shield coming from outside attacks and also survey unusual admittance along with utilization issues. Additionally, you should deploy along with put in force strong hallmark as well as gain access to handle plans to be sure safe VANET functionality for the users. Alternatively, indicator nodes possess minimal electricity and also producing assets. As a result, regular security systems that eat a lot of assets are not desirable. One example is, encrypted sheild/decoding procedures call for high computational might in addition to authenticating every correspondence along with user besides demands high interaction fill. These kind of challenges improve the need to produce detailed and also safe answers in which accomplish bigger security, while maintaining attractive community overall performance and also appropriate energy usage when necessary. Middleware work must focus along creating and also adding protection functions from the primary levels involving software program layout, thence efficiently accomplishing unlike security demands like secrecy, authentication, strength, gall, as well as availableness. A number of operate in investigation probable security measures techniques for SOM on the whole can be acquired [3]. However, to a greater extent attempt in order to conform most of these security benefits to the unique wants associated with VANET is necessary.

\subsection{Quality of Service}

Quality of service can be an over-used expression having numerous symbolism and perspectives through different inquiry as well as expert communities. Inwards VANET, we could view QoS through a couple of viewpoints: application distinct and community. The first kind refers to QoS details specific towards the software, for instance sensing element lymph gland measuring, deployment, and reporting and also variety of productive warning nodes. The latter is the term for exactly how the actual assisting connection network could fulfill app inevitably while effectively using multilevel sources such as data transfer usage as well as electricity consumption. Regular QoS mechanisms used in sent communities aren't equal intended for VANET as a consequence of difficulties including learning resource disadvantages along with dynamic topology.

Thus, middleware should provide new mechanisms to help keep QoS in excess of a prolonged period of time and in some cases alter alone if the expected QoS plus the point out with the diligence improvements. Middleware ought to be intended according to tradeoffs among functionality prosody for instance network ability or even throughput; facts supply delay, and energy consumption.

\subsection{Is Integrated to Programs}

Almost all sensing unit network purposes consist of warning or maybe RFID communities and also their helping software factors that being incorporated with other programs for instance endeavor as well as world-wide-web methods. This kind of integrated is essential to achieve the meant desired goals of utilizing detector communities earlier due to the fact collected files are generally unproductive unless it truly is inclined to the planned focus on to help reach the final aim. Middleware can certainly ease which integration by allowing company as well as vane systems for you to smoothly and properly connect with, command and collect the actual necessary info from VANET or maybe RFID networks. This call for delivering some connects that will facilitate the actual plug-in in addition to corroborate your traded selective information involving the VANET in addition to the additional organization.

\section{SOM REQUIREMENTS PERTAINING TO VANET}

SOM intended for VANET is essential to guide several, or else difficult to incorporated, benefits inside the SOC model. These kinds of functionalities consist of not only the useful demands, but also these no-functional needs in which diverse services may need to offer. In numerous request domain names, providers give you a set of distinct features using the required diligence logic that is to be different for each support as well as each and every request. On the other hand, those solutions and also companies must also assistance numerous usual benefits which might be generally irrelevant towards principal software. Intended for instance, about solutions have to service assistance registries and also breakthrough things and they will also need to provide approximately level of abstract entity to hide the root setting and implementation details. On top of that, much SOC software should assist some stages of dependableness, execution, security, as well as QoS. These can be supported making offered by having a usual middleware program instead of having to add all of them into every single overhaul and also request developed. Normally, depending on the observations, a SOM for VANET ought to assistance a number of specifications (down the page). The very first several are the essential as well as cosmopolitan overhaulorientated needs that need to be readily available in different overhaul-orientated system. All of those other specifications are usually unplanned with the characteristic of this VANET environment on one hand along with the challenge regarding implementing along with operating programs thereon environs on the other guitar. Certain requirements are:

i. Runtime assistance regarding products and services deployment and also rendering: seeing that a VANET can be regarded as a couple of solutions offered for assisting many apps, SOM usually supplies elements in order to release, weight, as well as implement these facilities. 
ii. Support pertaining to service buyers to uncover and employ authorized services: SOM should enable consumer purposes to learn and use listed providers. SOM should permit the integration among programs and also listed companies.

iii. Support visibility to be able to client purposes: SOM should enable node purposes in order to transparently employ available services without subjecting the skills enactment information maybe in many circumstances it's detailed components areas.

iv. Abstractions to cover your heterogeneity regarding implicit in sensing unit surroundings: wholly heterogeneousness information on VANET hardware as well as community needs to be disguised through the software. SOM has most likely furnished large-flat connects to use VANET means without necessitating the applying programmers to package direct with all the heterogeneity. This particular prerequisite details the actual heterogeneity as well as circle business issues talked about in the last segment.

v. Configurable providers: This requirement is usually to assistance remedy these hardware methods along with app expertise troubles talked about in the previous area. SOM thought to provide things with regard to customer applications to configure VANET solutions to satisfy distinct request specifications for instance QoS, security measures, or reliability. Setting up VANET typically calls for dealing with facts as well as details of several ironware in addition to transmission factors. Undertaking this kind of intended for establishing QoS as an example would have been a real sophisticated task for unconstipated users. Even so, SOM can offer mechanisms for use very easily through purchaser software for you to configure VANET pertaining to distinct program demands.

vi. Documentation with regard to self applied-business mechanisms: this could include home-by components this sort of ego-administration, personal-therapeutic, homesetting, vehicle-finding, personal-variation, and egomarketing and services information providers. VANET are usually active spread circumstances in which nodes may crash in addition to new nodes be able to be extra when. Moreover, mobile phone nodes with a few companies could be accessible for quite a while. The supply of services over these nodes can be powerful. For that reason, SOM must documentation home-management, auto-breakthrough discovery, and vehicle-alter, self optimization, as well as automobile-alter mechanisms with regard to useful by using accessible services. By way of example, if a node supplies a assistance pertaining to perception some attribute the actual SOM should discover that support as well as allow the customer purposes make use of that once they need it. Any time that guest fails, SOM must mechanically switching the programs to some equivalent assistance you can buy upon a different client within the community. What is more, the actual middleware really should be in a position to give notice the actual software if almost nothing matching their particular needs is now offered. This kind of prerequisite assists in solving this scalability, heterogeneity, net corporation, along with request information difficulties stated in the earlier part.

vii. Interoperability using a number of gadgets: This particular need facilitates clear up your heterogeneity difficulties described in the earlier section. Approximately sensing unit applications involve variety of devices to become run. SOM regarding VANET could be design and style to become interoperable with various products such as products with various types of detectors, RFID, in addition to mobile phone nodes make it possible for uncomplicated application development and operations.

viii. Effective coping with of large sizes of knowledge and also in high spirits communication lots: This demand helps with fixing your minimal electronics resources along with network firm issues talked about in the earlier area. Several VANET applications contain large volumes of data and also higher communicating lots. Because VANET have limited sources, SOM should correctly and also carefully take care of that weight as a result of business-offs among application specifications with regards to reliability, data transfer rate, postpone, and intake, being unfaithful.

ix. Safeguarded conversation along with setup: as VANET are currently being wide started with internet domain names that involve sore and also essential details, risk-free transmission and also rendering gets a vital aspect in SOM pertaining to VANET. SOM must provide mechanisms for you to secure the employment in addition to trading operations involving VANET solutions. Many transmission as well as delivery for encouraging these services should be collateralized.

x. Assist intended for QoS demands: a sizable choice of VANET applications get QoS specifications. Systems are usually required to configure along with gratify these kind of demands inside VANET situations. Illustration of QoS prerequisites within VANET can be revealing an important examining within a specific time period in addition to within a sure blunder level. In some predicaments, this QoS needs will come from numerous software. SOM for VANET has most likely furnished standard interfaces to be able to set up this QoS needs for most of these programs as well as guaranteeing attaining these types of requirements.

xi. Assist pertaining to integrating along with other techniques: as VANET normally tend not to operate throughout remote location, SOM make it possible for the particular integrating regarding VANET to devices for instance business or net methods. e.g., approximately internet purposes depend upon VANET for his or her recent details including info on varying weather conditions, shipments spots, and dealings problems. In such cases, SOM must allow in which integrated, in a way that most of these apps can easily fulfill their set goals.

Besides these kinds of specifications, there are more superior as well as particular specifications which might be meant for some state-of-the-art along with specialized programs. One of them these requirements is definitely help intended for range of motion. For just about any regarding software with fluid sensing unit nodes inside the VANET. An additional qualification example is actually assistance intended for framework cognizance. SOM can supply several things with regard to software to simply along with expeditiously purpose concerning as well as reply to it is environmentally friendly contexts.

Many of these talked about specifications will also be essential for some SOM applied regarding other types associated with techniques such equally Power company and also business systems. With the key characteristic in which distinguishes VANET environments via people is the fact all these requirements must possibly be intended along with executed upon minimal working out, communication, recollection, and energy means. Your middleware companies 
in the SOM have to be light in weight and very productive throughout means use.

In general, scientists suggest SOM techniques very best suited for their implicit in conditions. As a result, numerous of these techniques whitethorn produce many of the requirements described previously mentioned, but most advisors usually do not help wholly demands. Within the pursuing incision, this site offers a discussion regarding approximately solutions.

\section{SOM APPROACHES PERTAINING TO VANET}

Even though doing your inquiry throughout the different techniques experts in addition to experts are working upon, we got over a substantial swimming pool of illustrations in SOM pertaining to VANET. Several solutions are getting examined with the investigation level while any few are truly used in practice. The main concern to handle right here is the variety in addition to heterogeneousness from the receptors used along with the systems linking these. Numerous issues were addressed by this category of methods just like coping with building complex as well as/or constant inquiries, information admittance, gathering or amassing and also management, overhaul publication, breakthrough discovery along with arrangement, collection operations, actual-period access to info in addition to solutions, and also constrained sources. The following is usually a small summary of the chosen approaches:

\subsection{SStreaMWare}

SStreaMWare [33] is a detector arbitrator SOM that provides a new question plan to entry information issued by heterogeneous alerts. The leading goal is to give you a mix of both option concentrating in sensing unit heterogeneity direction, scalability, and difficult dilemma rating. The principle components of SStreaMWare would be the detectors, the proxy servers and adapters, the actual gateways, and the particular controller web sites. It is buildings include 3 querying products and services plus a breakthrough/presenting services. The particular Inquiry Assistance is actually stationed for the management web site and is particularly in charge of inquiry evaluated. The Trip Overhaul will be implemented upon just about every gateway and gives a question overhaul to the detectors been able aside that will portal. The actual Proxy Program is actually deployed at worried gateways and provides solutions into a certain form of detectors. The Lookup Service is designed for services development as well as presenting within add-on to be able to handling the overhaul computer system registry.

\subsection{USEME}

USEME can be a support-orientated fabric to formulate instant indicator as well as actor or actress networks (WSAN) purposes. Throughout [14] some sort of middleware to aid, it's introduced. The main employment on the middleware is always to grip this book as well as discovery of web services; transmission associated problems, party management, as well as true-time difficulties. The particular computer architecture involving this middleware can be split into a few magnetic core factors including setup, publishing and breakthrough, conjure and communication, genuine-occasion limitation operations, along with collection supervision. Various guidelines like output deadlines and also breakthrough consistency may be improved by way of it configuration constituent. This distribution and also finding element monitors possible companies. The supplication and also connection component protects converting your distant commands completed through software computer programmers directly into transmission packages among remote control nodes. The particular real time restraint management portion handles difficulties such as environment your priorities intended for actions, specifying utmost carrying out time for any control, and also establishing time intervals intended for monthly activities. To obtain scalability in addition to productivity, the middleware types groups of your nodes of which share frequent behaviors in addition to immediately controls the employment of products and services provided because of the groupings using the collection direction aspect.

\subsection{SensorWeb 2.0}

To support outside Geospatial Range (OGC) efforts, the particular Cloud Calculating along with Sent out Systems (CLOUDS) Research lab in the School connected with Melbourne (http://www.gridbus.org/sensorweb/), will work on the SensorWeb labor (NICTA Available Warning World wide web Architecture (NOSA)) [17,31]. On this task, they covers a SOM to guide info selection in addition to tricks crosswise heterogeneous sensing element sites. The key issue is always to maintain continuous approach and employ involving sensing element information over extended time frames.

NOSA gives service-primarily based entry to as well as administration associated with detectors. It helps combine sensing unit systems and sent out calculation tools including SOA and also Grid Calculating. This key positive aspects usually are: Information science can easily be moved from detector networks for the backend spread techniques, particular person sensing element systems could be coupled jointly since solutions which could end up being documented, discovered, in addition to accessed simply by distinct clientele utilizing a homogeneous communications protocol. The principle components explained regarding NOSA consist within four levels: textile, services, growth, and request. An excellent the subsequent warning solutions: (one particular) detector notification, series, in addition to observation; (a couple of) files variety, collection, as well as archival; (three or more) sensor co-ordination and also facts control; (some) faulty detector info a static correction and also administration; in addition to (five) warning construction along with directory program.

\subsection{OASiS}

OASiS [5] is definitely a target-centrically, program-driven, ambient aware indicator multilevel purposes growth product. It specifies your plausible elements necessary to assist a large number of VANET dataflow programs. It specifies the programming platform that will facilitates separation associated with problems pertaining to program development by having a multilayer growth course of action. These key warning network functionalities usually are enclosed while middleware companies which includes support find, service graph make up, failing discovery, along with client supervision. Using the Haven platform, app builders do not demand just about any expertness in sensor multilevel programming. This framework address approximately designing troubles with regard to networked stuck warning methods aside promoting dynamic overhaul breakthrough along with setting, heterogeneousness, actual-planet plug-in throughout application design, demands operations, data collection as well as options regarding equally programunique and net QoS demands. The particular Haven middleware incorporates services like Guest Boss, Thing 
Supervisor, and also Powerful Support Configurator and gives the actual help towards software solutions.

\subsection{B-VIS}

B-VIS (Burapha Car-Base Organization) [45] is usually an A littl aiimed at facilitate the utilization involving dispersed RFID sensor networks pertaining to car-dependent software. It helps true-occasion vehicle fade monitoring as well as handle and also sustains road visitors keeping track of programs. Additionally, it provides a coding modelling for builders to be able to get back, in real-period, the actualinformation equanimous because of the RFID sensing unit cpa networks. This particular cuts down on difficulties on the heterogenous communication level in addition to files places. This B-VIS SOA is dependent on start procedures and reduce combining between software package along with the computer hardware parts that will assist in conversation. There's two main levels, the actual W-Baseborn Entry level as well as the Endeavor Software Waiter Coating.

\subsection{MiSense}

MiSense [30] is usually a program-focused aspect-dependent middleware built to support allocated warning apps with assorted overall performance prerequisites. It cuts down on complexity aside impacting on any construction in addition to the actual portion product available as composability rules and also oblation nicely-outlined overhaul-certain connects for the remaining organization. MiSense supplies a welldescribed content material-primarily based submit/register services, but in addition permits the approval artist in order to conform this services. The particular middleware is definitely partioned inwards cellular layers: Connection Stratum, which gives some sort of good-outlined content material-primarily based post/register service termed MiPSCom, allowing the application custom for you to modify this overhaul by designing orthogonal options around the transmission communications protocol pieces with regard to subscription and appraisal shipping and delivery. The normal Providers Level, which provides companies common to different types of software, such as information accumulation, event detection, network topology supervision, and course-plotting. The actual Domain Coating, which is designed to support domain specific needs, including data optical fusion and particular facts semantic support allowing the production of request-related to information coming from raw data control. The actual programming screen offers a set of capabilities that will enable the person to control and method the actual warning multilevel all together multilevel with distinct useful features without distressing approximately your comprehensive keeping calculations along with communicating.

\subsection{SOMDM}

The experts propose a message driven SOM to service ambient mindful Wi-Fi sensor cpa networks (VANET) [39]. One of many crucial things to consider inwards VANET is usually to preserve digesting cost to do business to start. As a result, the particular suggested design and style switches into middleware and also assistance-oriented architecture breakthroughs for you to greater manage your producing cargo. This gives the particular VANET for you to deal with the true-period need for question control along with unlined integrated, in addition to rendering regarding procedure reasoning. This layout besides contains any info filtration system for filtering events of great interest, which cuts down on the force on your processing elements by using a presentment support. This particular middleware includes various factors: the object rule needs nodes of the VANET to follow along with the particular normal development product; The actual Data Filter Container stands between convention as well as especial(a) activities where by typical data are usually directed toward any data source in addition to remarkable functions are generally submitted to your Message Line up; The Messaging Machine retailers this mail messages in a queue up; This Texting Pieces will likely be notified by simply the message entrance in the message queue; The DBMS must be used to help shop the information coming from the DataFilter Field; The actual Managing Sprint- Table will allow the particular owner in order to set up the middleware; The organization Is intergrated Structure allows various factors, companies, legacy methods, messaging hosts, and consumers connects to help communicate; and The Consumer Connects make it possible for buyers for you to inquiry this receptors in addition to watch the particular admonition talk about as well as the parts required to complete $\mathrm{M}$ functions.

\section{APPLICATION INEVITABLY}

VANET aren't basic function systems like the World-wideweb, although VANET applications are distribution over a mother board selection, they are generally started intended for quite certain purposes. Your programs the VANET can be focused on would enormously have an effect on the selection of middleware along with companies of which support the exploitation and also trading operations on the app. VANET Applications could be split into various categories that want distinct companies from your supporting middleware. Using the group of the appliance, the desired services tend to be motivated. The probable categories are generally: range, accessibility, criticalness, intricacy, visitors coarseness, and use connected with stand alone vs built-in applications.

\subsection{Degree}

VANET may be used in a tiny size using couple of adjacent warning nodes as with system detector systems or in a sizable measurement together with a huge number of detector nodes allocated spanning a substantial region as in field surveillance. From the second scenario, there is excessive requirement of middleware that delivers personal-business services to handle the issues of faithfully, productivity, and also manageability with the network. Convenience: VANET can be used within accessible regions these as in this production area or perhaps in inaccessible areas for instance monitoring subaqueous pipelines in which expand regarding thousands of kilometers. From the inaccessible situation, the advantages of middleware that delivers strength-productive, configurable, as well as personal-sorted services will be in high spirits. This can be caused by difficultness of updating nodes when ruined as well as depletes electrical power. The particular shape and administration of the type of net is definitely additionally a difficult problem which can be comfortable with all the universe involving configurable and self-organized services. In addition, it is important to possess deployment service that permits the productive installation of brand new and also up to date software program.

\subsection{Criticality}

VANET can be employed intended for uncritical application domain names including habitat realizing or perhaps very crucial domain names that in high spirits safety in addition to QoS for transporting believed info including throughout infrastructure overseeing along with field surveillance. Therein type of decisive apps, certain requirements pertaining to security measures as well as QoS providers are 
generally large and may possibly be offered by the particular middleware.

\subsection{Complexness}

VANET situations are usually elementary having organised sent out homogenous nodes as in VANET regarding checking buildings; or even intricate with good dynamic unstructured sent out heterogenous nodes. In the second option scenario, generally there is often a solid desire for solutions that provide great abstractions to cover the particular heterogeneity from the fundamental sensor conditions. These types of services really should be besides interoperable that has a number of equipment. This middleware companies ought to be see-through on the node purposes. Moreover, complexity is elevated with extremely dynamic configurations, therefore we want efficient learning resource breakthrough discovery services in addition to selfconstitution solutions to help effectively use the offered assets in order to meet the particular apps needs similarly and to expand everything from the multilevel on the other half. Site visitors coarseness: several indicator software only need to obtain information about exceptions say for example a immediate alternation in heat range, demand, as well as activity. One of these of such purposes will be a VANET for monitoring woods with regard to shoots. VANET is only going to survey if you experience a sudden boost in your temp (seeing that sign connected with fireplace nearby). This kind of purposes will probably produce low transmission dealings and also would not really demand a high need around the devices resources. About contrary, some software rich person steady stream devices such since camcorders and audio recorders that to deliver ongoing water ways of knowledge thence making high transmission stress on this system. In cases like this, there exists substantial need for middleware services which could correctly handle in high spirits site visitors as well as change your multilevel functions pertaining to optimum functionality. By using standalone vs . structured software: VANET may be formulated in addition to fitted like a separate app that will not ought to be included for some other systems. Within this case, you will find generally zero demands on the style as well as setup. Then again, they will must always be integrated to systems including company along with world wide web applications. In this case, there exists a higher requirement of any middleware program that enables that plug-in efficiently in addition to quite easily.

\section{DISCUSSION}

SOM programs look at VANET like a group of solutions distributed along the multilevel having properly-outlined connects. These facilities can be easily identified in addition to used from the SOM aside VANET apps. The position connected with SOM pertaining to VANET is not only to look after sensible properties (like connection, program design, breakthrough discovery as well as conjuration, party direction, and real-occasion functions) but additionally to compliment not for-functional components (like scalability, service consistency, service flexibility, interoperability, along with QoS guarantee). With Desk just one, many of us sum it up the different SOM approaches pertaining to VANET and also recognize the particular needs to each one approaching fulfills.

In achieving this SOM should employ possibly metadevelopment dialects or maybe knowledge domain-unique 'languages'. Such a project continues to be consumed in the situation and services information-
Focused Situation-Aware middleware that creates using it's have produced area-distinct speech expressing your situation based mostly policies. The particular ruler supervision strategy in addition to notification program with the aid of sector-distinct dialect becomes helpful especially in circumstance and services information deployment. Given that two interactive get-togethers mightiness communicate by means of multiple routes, discovering the right joining way to offer confirmed conversation can be a important problem to cope with, particularly in SOC as this appreciably impacts the particular QoS [18]. What is more, due to the fact SOM with regard to VANET will be based upon providers of heterogeneous nature, the latency these services is just about the variables that directly affect the effectiveness. To be able to improve efficiency, the particular latency from the services inevitably to be improved. Such an betterment may be possible by simply adding UDP-based mostly SOAP move as an alternative to HTTP primarily based transportation [32]. Nonetheless, a standard SOM, which usually addresses big degrees of emails and also consequence notices caused by legion(p) providers in addition to explains to you them among unique working components, must have a reliable program in place in which assures the best flowing associated with appropriate in addition to timely messages. This kind of may be accomplished utilizing event managing tactics which carry out unique mechanisms to be sure QoS along with stability within VANETs. With QoS, the particular bad result of complications for instance conversation over-crowding and connection faults might be significantly diminished. Some of the interviewed tasks structured QoS contained in the recommended SOM with regard to VANETs. Examples of these types of initiatives are Retreat [4], SensorMW [5], and also ubiSOAP [13]. While these types of assignments used many QoS things such as best offered system option in line with the QoS demands and also a contract settlement based on assistance amount contracts, different state-of-theart QoS mechanisms forVANETs[16] can be included along with SOM. Additionally, fault managing elements intended for VANETs [43] can be built-in with SOM. Furthermore, SOM functions the task and services information orchestration.

Splitting the applying common sense from your organization common sense by way of a middleware accomplish assists in these kinds of pastime. Any middleware in line with the notion of Model Motivated Computer architecture (MDA) in addition to SOA is released inwards [44]. SOA design and style designs tend to be pattern strategies that assist triumph over popular limitations within having this arranged goals and also gains associated together with SOC [29]. However, the development of middleware alternatives making use of SOA designing styles is still at its birth. Researchers applied engineering for example internet providers and also Peer-topeer to development SOM. In terms of the requirements discovered in Section 4, we all observed that will no SOM strategies outlined includes most advisors. In truth, hardly any of which address actually half of these prerequisites. But, it may be achievable to look at this like a typical train considering that most SOM solutions are created to work out a particular pair of troubles in order to concentrate on i sort or even class of software areas. Thus, it'll be healthy to locate solely those people requirements which have been almost all needed in which knowledge domain. Just one useful paying attention we furthermore announced is the fact that about no solutions learnt tackles the safety difficulties as part of the SOM requirements. Most of us acknowledged this just as one of import problem intended for SOM in particular pertaining 
to VANET because of the particular limitations in VANET methods. Many assaults and difficulties are potential using a VANET as well as, although not limited by, eavesdropping, criminal nodes, self-denial and services information assaults, refutation files, in addition to redirecting people to interrupt trading operations. This particular simply leaves all of us that have a big candid issue regarding the incredible importance of including safety measures from the SOM. As it's wellknown, security measures is significant for virtually any form of dispersed circumstances in addition to SOC intended for VANET isn't any different. A different one of the primary challenges connected with putting into action in addition to running VANET compared with other kinds regarding systems is handling the restricted sources associated with calculation, sensing, communication, and to satisfy application demands on the one hand and maximize the life of the net on the other instrument. Useful resource managing in VANET is definitely not really a not important concern specifically in heterogenous in addition to powerful situations. This particular operations may well be more difficult by having a number of purposes fighting over the obtainable solutions within the special VANET resources. Although SOM websites make simpler the development strategy of VANET applications, these services likewise need very careful design to satisfy app prerequisites along with lower limit resource ingestion. Advanced optimization approaches are required with regard to assets breakthrough discovery, part, and also operations. These kinds of approaches must also follow power-productive. A few attempts had been carried out ply learning resource allocations optimisation methods of wireless detector sites generally speaking $[20,27]$. Additionally, a new middlewarebased resourcefulness managing pertaining to VANET originated inside [47]. In this particular work, the particular VANET can be put into many groupings involving nodes. A clustering is often a list of spatially next nodes that will work to evaluate specific phenomena. Cluster a VANET allows the particular middleware to help efficiently manage means with minimum amount electricity usage. This particular middleware offers a personal car type together with ii tiers: cluster stratum in addition to useful resource management coating. The particular group layer is answerable to forming some sort of bunch regarding detector nodes that are around the objective phenomena. The particular learning resource direction level is responsible for the actual storage allocation and edition connected with sources such that unique app requirements for example QoS, reliability, and security may end up being fulfilled. The work was made pertaining to neoSOM pertaining to VANET. Withal like architectures works extremely well by alterations for SOM pertaining to VANET. This SOM is usually built to include a few tiers associated with services since proven in Fig. a single. These tiers usually are clump companies layer, resource administration solutions level, in addition to sophisticated services covering providing you with strong companies pertaining to the particular software for instance QoS, security, discovery, stability and so forth. To each one stratum offers companies for that top levels. Pertaining to illustration, apps can use this state-of-the-art companies coating to meet his or her needs. The particular innovative companies layer use this source direction level for you to proficiently utilize available helpful information on executing various solutions simply by delivering useful sources allocation along with adaptation mechanism purposes On top of that, your useful resource administration coating works by using companies provided by the particular clustering bed to help successfully take care of groups' means.

\section{DECISION}

Within this newspaper, most of us talked about the overall prerequisites regarding SOM for VANET and reviewed several adviser methods within the discipline. SOM offers middleware services for assorted SOC domain names and is also helpful for different environments for instance VANET. Today experts as well as users would rather utilize the actual SOM approaching in excess of conventional middleware because of its characteristic regarding proficiently controlling heterogeneous pieces using often combined companies. The strategies reviewed take a few parallels inside the impression that advisors agree with one common target: to be able to properly make, release, uncover, and also utilize companies. Nonetheless, the methods applied were likewise drastically different within the direction they satisfy that objective. In addition, the prospective benefits of your solutions are different exactly where targets similar interoperability, QoS assistance, authentic-moment documentation, heterogeneity support, scalability, and context-cognizance could happen. Most of us outlined many SOM intended for VANET projects as well as underlined the major ambitions in addition to approaches besides xactly how properly these people match the cosmopolitan SOM prerequisites. SOM is actually destined to grip the particular book and development regarding vane services, communication, as well as effective as well as dependable celebration supervision amid unique services. Furthermore, SOM solutions have to service useful controlling in the heterogenous sources and also the uses of the VANET applications. Moreover, due to should swap substantial quantities of web data, it is necessary to get a trustworthy along with useful organization constantly in place to make certain a uninterrupted flow of accurate and also well-timed emails. It is usually important to retain appropriate numbers of management, safety measures, as well as dependability over the SOM that can essentially think about the particular purposes making use of it. In the end, to further increase the SOM we must possess dynamic, adaptative as well as automobile-configurable architectures intended for efficient integrating and also reuse.

\section{REFERENCES}

[1] Abangar H, Barnaghi P, Moessner K, Nnaemego A, Balaskandan K, Tafazolli R (2010) A service oriented middleware architecture for wireless sensor networks. In: Proceedings of future network and mobile summit conference

[2] Al-Jaroodi J, Mohamed N, Jiang H, Swanson D (2003) Middleware infrastructure for parallel and distributed programming models on heterogeneous systems. IEEE Trans ParallelDistributed Syst 14(11):1100-1111

[3] Al-Jaroodi J, Al-Dhaheri A (2011) Security issues of serviceoriented middleware. Int $\mathrm{J}$ Comput Sci Netw Secur (IJCSNS) 11(1):153-160

[4] Amundson I, Kushwaha M, Koutsoukos X, Neema S, Sztipanovits J (2006) OASiS: a service-oriented middleware for pervasive ambient-aware sensor networks. In: Pervasive and mobile computing journal on middleware for pervasive computing, October 2006

[5] Anastasi GF, Bini E, Romano A, Lipari G (2010) A service oriented architecture for QoS configuration and management of wireless sensor networks. In: The 15th IEEE international on emerging technologies and factory automation. Bilbao, Spain, September 2010 
[6] Anke J, Müller J, Spieß P, Weiss Ferreira Chaves L (2006) A service-oriented middleware for integration and management of heterogeneous smart items environments. In: Proceedings of $4^{\text {th }}$ MiNEMA workshop, pp 7-11, July 2006

[7] Avilés-López, E, García-Macías, JA (2009) TinySOA: a service oriented architecture for wireless sensor networks. Service Oriented Computing and Applications. 3(2):99-108 (Springer)

[8] Bai Y, Ji H, Han Q, Huang J, Qian D (2007) MidCASE: a service oriented middleware enabling context awareness for smart environment. In: Proceedings of international conference on multimedia and ubiquitous engineering (MUE'07)

[9] Bichler M, Lin KJ (2006) Service-oriented computing. IEEE Comput 39(3):88-90

[10] Blaya J, Demeure I, Gianrossi P, Lopez P, Navarro J, Meyer E, Pelliccione P, Tastet-Cherel F (2009) POPEYE: providing collaborative services for ad hoc and spontaneous communities. Serv Oriented Comput Appl 3(1):25-45 (Springer)

[11] Britton C (2001) IT architecture and middleware: strategies for building large, integrated systems for building large, integrated systems. Addison-Wesley, Reading

[12] Canete E, Chen J, Diaz M, Llopis L, Rubio B (2009) A service oriented middleware for wireless sensor and actor networks. In: Proceedings of 6th international conference on information technology: new generations. Las Vegas, USA

[13] Caporuscio M, Raverdy P-G, Moungla H, Issarny V (2008) ubiSOAP: a service oriented middleware for seamless networking. In: Proceedings of 6th ICSOC

[14] Caporuscio M, Charlet D, Issarny V, Navarra A (2007) Energetic performance of service-oriented multi-radio networks: issues and perspectives. In: Proceedings of WOSP

[15] Chan S, Kaler C, Kuehnel T, Regnier A, Roe B, Sather D, Schlimmer J, Sekine H, Walter D, Weast J (2006) Devices profile for web services, available at http://schemas.xmlsoap.org/ws/2006/ 02/devprof/, February 2006

[16] Chen D, Varsheny PK (2004) QoS support in wireless sensor networks: a survey. In: Proceedings of international conference on wireless networks (ICWN '04), pp 227-233, Las Vegas, Nev, USA, June 2004

[17] Chu X, Kobialka T, Durnota B, Buyya R (2006) Open sensor web architecture: core services. In: Proceedings of 4th international conference on intelligent sensing and information processing. Bangalore, India, December 2006

[18] Costa N, Pereira A, Serodio C (2007) Virtual machines applied to WSN's: the state-of-the-art and classification. The second international conference on systems and networks communications (ICSNC 2007), August 2007

[19] Delicato F, Pires P, Pinnez L, Fernando L, da Costa L (2003)A flexible web service based architecture for wireless sensor networks. In: Proceedings of 23rd international conference on distributed computing systems workshops, 2003

[20] Giannecchini S, Caccamo M, Shih C-S (2004) Collaborative resource Allocation in wireless sensor networks. In: Proceeding of Euromicro conference on real-time systems (ECRTS'04), pp 35-44, June/July 2004

[21] Gibbons P, Karp B, Ke Y, Nath S, Seshan S, Res I, Pittsburgh P (2003) Irisent: an architecture for a worldwide sensor web. IEEE Pervasive Comput 2:2233

[22] Gnawali O, Jang K, Paek J, Vieria M, Govindan R, Greenstein B, Joki A, Estrin D, Kohler E (2006) The tent architecture for tiered sensor networks. In: Proceedings of the 4th international conference on embedded networked sensor systems, pp 153-166, 2006

[23] Golatowski F, Blumenthal J, Handy M, Haase M, Burchardt H, Timmermann D (2003) Service-oriented software architecture for sensor networks. In: Proceedings of international workshop on mobile computing, IMC 2003, pp 93-98, June 2003

[24] Groba C, Braun I, Springer T, Wollschlaeger M (2008) A serviceoriented approach for increasing flexibility in manufacturing. In: Proceedings of IEEE international workshop on factory communication systems, 2008

[25] Gu QJ, Lago P (2009) Exploring service-oriented system engineering challenges: a systematic literature review. Serv Oriented Comput Appl 3(3):171-188 (Springer)

[26] Hadim S, Mohamed N (2006) Middleware for wireless sensor networks: a survey. In: Proceedings of the first international conference on communication system software and middleware (COMSWARE 2006), IEEE, New Delhi, India, January 2006

[27] Hadim S, Mohamed N (2006) Middleware challenges and approaches for wireless sensor Networks. IEEE Distrib Syst 7(3)

[28] Hadim S, Al-Jaroodi J, Mohamed N (2006) Trends in middleware for mobile ad hoc networks. Invited paper in the J Commun 1(4):11-21

[29] Introducing SOA Design Patterns (2008) SOA world magazine, 8(6), June 2008

[30] Khedo KK, Subramanian RK (2009) A service-oriented component- based middleware architecture for wireless sensor networks. Int $\mathrm{J}$ Comput Sci Netw Secur (IJCSNS) 9(3):174-182

[31] Kobialka T, Buyya R, Leckie C, Kotagiri R (2007) A sensorweb middleware with stateful services for heterogeneous sensor networks. In: Proceedings 3rd international conference on intelligent sensors, sensor networks and information processing (ISSNIP 2007). Melbourne, Australia, December 2007

[32] Lai KY, Phan TKA, Tari Z (2005) Efficient SOAP binding for mobile web services. In: Proceedings of LCN

[33] Levent G, Claudia R, Cyril L, Andre B,VincentO(2008) SStreaMWare: a service orientedmiddleware for heterogeneous sensor data management. In: Proceedings of ICPS, Sorrento, Italy, July 2008 
[34] Lipphardt M, Neumann J, Groppe S, Werner C (2008) DySSCo-a protocol for dynamic self-organizing service coverage. In: Proceedings of the 3rd international workshop on self-organizing systems, pp 109-120, 2008

[35] Liu X, Wang Q, Sha L, He W (2003) Optimal QoS sampling frequency assignment for real-time wireless sensor networks. In:Proceedings of the IEEE real-time systems symposium. Cancun, Mexico, December 2003

[36] Maia MEF, Rocha LS, Andrade RMC (2009) Requirements and challenges for building serviceoriented pervasive middleware.In: Proceedings of 2009 international conference on Pervasive services, London, United Kingdom, pp 93-102, July 2009

[37] Mohamed N, Al-Jaroodi J, Jawhar I (2008) Middleware for robotics a survey. In: Proceedings of IEEE international conference on robotics, automation and mechatronics (RAM 2008). Chengduo, China, September 2008

[38] Mottola L, Picco GP (2010) Programming wireless sensor networks: Fundamental concepts and state of the art. ACM Comput Surv, 2010. To appear. Available at: www.sics.se/ luca/papers/ mottola10programming.pdf

[39] Pandey K, Patel SV (2009) A novel design of service oriented and message driven middleware for ambient aware wireless sensor network. Int J Recent Trends Eng Acad Publ 1(1):313-317

[40] Papazoglou MP (2003) Service-oriented computing: concepts, characteristics and directions. In: Proceedings 4th international conference on web information systems engineering
[41] Papazoglou M,Traverso P, Dustdar S,Leymann F, KramerB(2006) Service oriented computing research roadmap. In: Proceedings of Dagstuhl seminar 05462 (SOC).

[42] Podolski I,RettbergA(2009)Aservice-oriented selfconfiguration middleware for autonomous sensor networks. Software Technologies for Future Dependable Distributed Systems

[43] Ruiz LB, Siqueira IG, Oliveira LB, Wong HC, Nogueira JM, Loureiro AAF (2004) Fault management in eventdriven wireless sensor networks. In: Proceedings of ACMinternational conference on modeling, analysis and simulation of wireless and mobile systems (MSWiM'04), pp 149-156, October 2004

[44] Sommer S, Buckl C, Knoll A (2009) Developing service oriented sensor/actuator networks using a tailored middleware. In: Proceedings of 6th international conference on information technology: new generations. Las Vegas, USA

[45] SriborriruxW, Kraipui S, Indra-Payoong N (2009) BVIS serviceoriented middleware for RFID sensor network. World Acad Sci Eng Technol 56:770-774

[46] Sugihara R, Gupta RK (2008) Programming models for sensor networks: a survey. ACM Trans Sensor Netw $4(2): 8: 1-8: 29$

[47] Yu Y, Krishnamachari B, Prasanna VK (2004) Issues in designing middleware for wireless sensor networks IEEE Netw Mag Special Issue 18(1):15-21 